\title{
Scalar and vector interactions of a composite spin-1/2 system
}

\author{
S. J. Wallace \\ Department of Physics and Center for Theoretical Physics, University of Maryland, College Park, Maryland 20742 \\ Franz Gross \\ Department of Physics, College of William and Mary, Williamsburg, Virginia 23185 \\ and CEBAF, 12000 Jefferson Avenue, Newport News, Virginia 23606 \\ J. A. Tjon \\ Institute for Theoretical Physics, University of Utrecht, 3508 TA Utrecht, The Netherlands
}

(Received 22 August 1995)

\begin{abstract}
Scalar and vector interactions, with the scalar interaction coupled to a composite spin-1/2 system so as to cause a shift of its mass, are shown to obey a low-energy theorem which guarantees that the second order interaction is the same as for a point Dirac particle, in which case the second-order interaction comes from $z$ graphs. Off-shell and contact interactions appropriate to the composite system cancel and this is verified in a model of a composite fermion. The low-energy theorem and its generalizations provide a justification for the use of the Dirac equation as it has been used in relativistic nuclear scattering and mean field theories.
\end{abstract}

PACS number(s): 24.10.Jv, 24.85.+p

\section{INTRODUCTION}

In a recent paper [1], a low-energy theorem has been formulated for the scalar and vector interactions of a composite spin-1/2 system. For a scalar interaction corresponding to a pure shift of the mass, the theorem shows that the secondorder interaction with the composite system is the same as for a Dirac particle interacting with an equivalent scalar potential. Including a vector interaction with only a time component does not change the result. This theorem, which holds in the limit of zero momentum transfer, provides definite conditions under which it is valid to treat a composite particle, such as a nucleon, as a Dirac particle.

Birse [2] has considered a more general combination of scalar and vector interactions and has shown from Lorentz invariance that, in addition to the model independent contribution to the second-order interaction discussed in this paper, there may be another scalar contribution that depends on polarization of the composite system. That scalar term is model dependent and will be considered in another paper [3].

The use of the Dirac equation in nuclear physics has been a subject of interest and debate in recent years. One of its outstanding successes is in elastic scattering of protons by nuclei $[4,5]$. Quite large scalar and vector interactions, which almost cancel one another, characterize the proton-nucleus interaction. Solving the Dirac equation with an attractive scalar potential and a repulsive vector one, each of magnitude about $300 \mathrm{MeV}$, produces a good description of spin observables at intermediate energies [6-10].

The new physics obtained by use of the Dirac equation, as opposed to use of the Schödinger equation, lies in the $z$-graph contributions. These are largest at zero momentum transfer and they decrease at higher momentum transfer. The significance of the $z$-graph contributions for a composite nucleon has been debated in the literature for a decade. Because they are difficult to understand from the point of view of perturbative QCD, it has been argued that the z-graph contributions should be suppressed by compositeness of the nucleon, even at zero momentum transfer [11,12]. A nonperturbative analysis [13] has produced the same conclusion in a simple $1+1$ dimensional theory. However, it has been argued that the same effects as are obtained from use of the Dirac equation for nucleons may be understandable in terms of quark $z$ graphs [14]. Arguments based on Lorentz invariance support the validity of the $z$-graph contributions for a composite system because they follow from a simple shift of the mass. The low-energy theorem clarifies this latter point of view.

In Sec. II, the general basis for the low-energy theorem is stated based upon considerations of Lorentz invariance. Emphasis is placed upon the case of a scalar interaction that shifts the mass and a vector interaction that shifts the energy. The balance of the analysis presented in this paper provides insight into the general result for a solvable model of a composite system. Details that were omitted in the earlier work [1] are provided and the previous analysis is clarified and extended to incorporate a suitable vector interaction. In Sec. III the solvable field-theory model of a composite fermion is introduced and the second order response to an external vector field is studied. It is shown how to arrive at the lowenergy theorem for this case. In Sec. IV the scalar interaction that generates a pure shift of the mass is developed and identities appropriate to the scalar interaction for the composite fermion model are derived. Several distinct contributions to the second-order interaction with the a combination of scalar and vector external fields are calculated in Sec. V, and it is demonstrated how cancellations take place which produce the simple result provided by the low-energy theorem. Conclusions and a discussion of the results are given in Sec. VI.

\section{CONSEQUENCES OF LORENTZ INVARIANCE}

Consider the case of a scalar interaction $S$ that corresponds to a pure shift of the mass and a time component of a 
vector interaction $V$ that shifts the energy. The form of the classical Hamiltonian determined by Lorentz invariance is,

$$
H=V+\sqrt{(M+S)^{2}+\mathbf{p}^{2}},
$$

where the vector and scalar potentials are taken to be spatially uniform. Expanding in $S$ to second order yields,

$$
H=\epsilon+V+\frac{M}{\epsilon} S+\frac{\mathbf{p}^{2}}{2 \epsilon^{3}} S^{2}+\cdots,
$$

where $\epsilon=\sqrt{M^{2}+\mathbf{p}^{2}}$. If $S, M$, and $\epsilon$ are noncommuting operators, care must be taken to maintain Hermiticity in developing this expansion. The momentum dependent repulsive potential term in Eq. (2.2) provides the main relativistic effect in proton scattering by nuclei at intermediate energies.

One obtains the same results from the Dirac equation with scalar and vector potentials. The energy expression may be reduced to the form,

$$
E \psi=\left(\epsilon+\mathscr{V}^{++}+\mathscr{V}_{\text {pair }}\right) \psi
$$

where

$$
\mathscr{V}^{++}=V+\frac{M}{\epsilon} S
$$

and

$$
\mathscr{V}_{\text {pair }}=\frac{\mathbf{p}^{2} S^{2}}{\epsilon^{2}[E+\epsilon-V+(M / \epsilon) S]}=\frac{\mathbf{p}^{2} S^{2}}{2 \epsilon^{3}}+\cdots,
$$

is the z-graph contribution. To second order it is the same as in Eq. (2.2).

The low-energy theorem considered here is the following statement. In the presence of a constant, scalar interaction which causes a mass shift $S$, the energy of a particle contains the repulsive term $\mathbf{p}^{2} S^{2} / 2 \epsilon^{3}$ without any dependence on the composite structure of the particle. The constant interaction implies the low-energy limit, i.e., the limit of zero energymomentum transfer. In this limit, a composite fermion may be treated as a Dirac particle interacting with an equivalent scalar potential. It should be noted that at nonvanishing momentum transfer this no longer holds in general. The inclusion of a suitable vector interaction provides a shift of the energy and this corresponds to the case of interest in nuclear physics. This paper provides a full account of the demonstration of the low-energy theorem based on a simple model Lagrangian.

The proof of the low-energy theorem rests on three simple points. (i) For any composite system, it is possible to define a scalar interaction which produces a pure shift of the mass. (ii) Similarly, it is possible in general to define a vector interaction which produces a pure shift of the energy. (iii) The energy of the composite system is then restricted by Lorentz invariance to take the form of Eq. (2.1) and the expansion of the energy contains the momentum-dependent repulsive term of Eq. (2.2).

Point (i) may be proved by an explicit construction, as follows. Consider a Lagrangian $\mathscr{B}$ that supports a compositeparticle bound state of mass $M$. Let parameters with dimensions of mass of the Lagrangian be scaled by a factor $\lambda$ and let any other parameters with dimensions be scaled accordingly, i.e., a parameter with dimension $1 /$ mass be scaled by $1 / \lambda$. Similarly scale all parameters generated by renormalizing the theory. The bound state mass will be shifted to $\lambda M$. Choose $\lambda=1+S / M$ to produce the desired shift of the bound state mass $M \rightarrow M+S$. The required scalar coupling is obtained by expanding the scaled Lagrangian about $\lambda=1$ in powers of $S / M$, and to first order one obtains $\mathscr{B} \rightarrow \mathscr{b}-\rho S$, where $\rho$ will be called the scale-breaking charge. The coupling term, $\rho S$, defines the interaction which shifts the mass.

In general, the scale-breaking charge, $\rho$, is proportional to the divergence of the dilatation current of the system. This may be seen from the following argument. A dilatation of all coordinates by the factor $\lambda^{-1}$, taken together with a scaling as described above of all parameters with dimensions by the factor $\lambda$, leaves the action invariant, i.e., $\int d^{4} x \mathscr{C}$ does not change. Thus the scaling of dimensionful parameters has the opposite effect of a dilatation. The system is not invariant in general under a dilatation and the change of the Lagrangian is proportional to the divergence of the dilatation current, $s_{\mu}$, which in turn is proportional to the scale-breaking charge as follows,

$$
\partial_{\mu} s_{\mu}=-M \rho
$$

Addition of a term $-S \rho$ to the Lagrangian is equivalent to a dilatation of coordinates by $(1+S / M)^{-1}$, or a mass scaling by $1+S / M$. This argument is based on the classical Lagrangian but it holds also at the quantum level provided that anomalous contributions, which are generated by renormalizing the theory, are included in the scale-breaking charge.

Point (ii) follows from coupling the system to an external field through the time-component of the total momentum. In practice, it often is sufficient to couple to the timecomponent of a conserved vector current. The low-energy theorem follows by point (iii) for a general composite system. Thus the repulsive term that is associated with $z$-graphs for a Dirac particle is guaranteed. This term is model independent in the sense that the second-order term is equal to the square of the first-order term, $(M / \epsilon) S$, times the kinematical factor $\mathbf{p}^{2} /\left(2 \epsilon M^{2}\right)$.

We now turn to a detailed proof of the low-energy theorem for vector interactions.

\section{INTERACTIONS WITH EXTERNAL VECTOR FIELDS}

In this section we will give a general discussion of how a composite system propagates in free space, and of how this propagation is modified by the presence of an external vector field which shifts the energy.

Consider a composite, spin-1/2 particle with total momentum $p$ which has a bound state of mass $M$. In the neighborhood of the bound state pole, we may assume the propagator takes the general form

$$
\mathscr{G}=\Omega G(p) \Omega+\mathscr{R}
$$

where $\Omega$ and $\mathscr{B}$ are dependent on the total momentum and the internal variables of the composite system but are regular at $p^{2}=M^{2}$. The bound state pole resides in the factor

$$
G(p)=1 /\left[1-p A\left(p^{2}\right)-B\left(p^{2}\right)\right]
$$




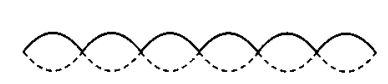

FIG. 1. Diagrammatic representation of the propagator in the bubble model.

where $A$ and $B$ are scalar invariants, depending only on $p^{2}$. This ansatz incorporates the fact that the bound state pole is not dependent on the internal coordinates and that it should involve a standard representation of the Lorentz group for spin-1/2.

A concrete example of the assumed structure is obtained from the following model Lagrangian,

$$
\mathscr{C}=\bar{\psi}(i \not-m) \psi+\frac{1}{2}\left[\partial_{\nu} \phi \partial^{\nu} \phi-\mu^{2} \phi^{2}\right]-g \bar{\psi} \psi \phi^{2}-\mathscr{L}_{\Lambda},
$$

where $\mathscr{D}_{\Lambda}$ consists of cutoff terms sufficient to remove divergences of the bubble graphs we consider, i.e.,

$$
\mathscr{C}_{\Lambda}=\frac{1}{2}\left[\partial_{\nu} \phi_{\Lambda} \partial^{\nu} \phi_{\Lambda}-\Lambda^{2} \phi_{\Lambda}^{2}\right]-g \bar{\psi} \psi \phi_{\Lambda}^{2}
$$

The quantum propagator for the composite spin-1/2 system with four-momentum $p$ is given by the sequence of bubble graphs as indicated in Fig. 1 and it is written as,

$$
G(p)=\frac{1}{1-\Sigma(p)}
$$

where $\Sigma(p)$ is related to the self-energy. In the "bubble model," the self-energy $\Sigma$ is the contribution of a single bubble graph and it can in general be expressed in terms of the interactions between the constituents of the composite particle. Its general form is restricted by Lorentz invariance to be

$$
\Sigma(p)=A\left(p^{2}\right) p+B\left(p^{2}\right)
$$

with two scalar functions $A$ and $B$.

For either the assumed general form of Eq. (3.1) or for the bubble model propagator of Eq. (3.5), the presence of a spin$1 / 2$ bound state of mass $M$ means that there is a pole in $G(p)$ at $p=M$. Thus the propagator can be expanded around $p^{2}=M^{2}$, giving

$$
\begin{aligned}
G(p) & =Z_{2}\left[\frac{1}{p-M}+\delta G(p)\right]=\frac{\Gamma(p) \bar{\Gamma}(p)}{p^{0}-\epsilon}+\delta g(p) \\
& =G_{0}(p)+\delta g(p)
\end{aligned}
$$

where explicit expressions for the renormalization constant $Z_{2}$, and the terms $\delta G$ and $\delta g$, which are nonsingular as $p^{0} \rightarrow \epsilon$, are worked out in the Appendix, and $\Gamma(p)=\left(Z_{2}\right)^{1 / 2} u(p)$, with $u(p)$ the positive energy Dirac spinor for an elementary fermion of mass $M$ :

$$
u(p)=\left(\frac{\epsilon+M}{2 \epsilon}\right)^{1 / 2}\left[\begin{array}{c}
1 \\
\frac{\sigma \cdot \mathbf{p}}{\epsilon+M}
\end{array}\right]
$$

which obeys the normalization condition, $\bar{u}(p) u(p)=M / \epsilon$.

To calculate the propagation of the composite system in an external vector field which shifts the energy, one may evaluate the propagator

$$
G_{V}(p)=\frac{1}{1-\Sigma(p-V)}
$$

where the shift $p-V$ in the argument of $\Sigma$ is shorthand for the shift $\left(p^{0}-V, \mathbf{p}\right)$. If the external field is weak, one may expand this propagator in a Taylor's series, giving

$$
G_{V}(p) \simeq G(p)-\frac{\partial G(p)}{\partial p_{0}} V+\frac{1}{2} \frac{\partial^{2} G(p)}{\partial p_{0}^{2}} V^{2}+\cdots,
$$

where the derivatives can be written

$$
\begin{gathered}
\frac{\partial G(p)}{\partial p_{0}}=-G(p) \Lambda^{0}(p, p) G(p) \\
\frac{1}{2} \frac{\partial^{2} G(p)}{\partial p_{0}^{2}}= \\
+(p) \Lambda^{0}(p, p) G(p) \Lambda^{0}(p, p) G(p) \\
+\frac{1}{2} G(p) C^{00}(p, 0) G(p)
\end{gathered}
$$

with vertex function $\Lambda^{0}$ and contact term $C^{00}$ defined by

$$
\begin{aligned}
\Lambda^{0}(p, p) & \equiv-\frac{\partial \Sigma(p)}{\partial p_{0}} \\
& =\Lambda_{0}^{0}+\left(p^{0}-\epsilon\right) \delta \Lambda_{0}+\sigma\left[\left(p^{0}-\epsilon\right)^{2}\right] \\
C^{00}(p, 0) & \equiv \frac{\partial^{2} \Sigma}{\partial p_{0}^{2}}=C_{0}^{00}+\mathcal{Q}\left(p^{0}-\epsilon\right) .
\end{aligned}
$$

The quantities $\Lambda_{0}^{0}, \delta \Lambda_{0}$, and $C_{0}^{00}$ are all evaluated at the on shell point where $p^{0}=\epsilon$, so that Eq. (3.12) expresses the vertex function and contact term as a power series in $p^{0}-\epsilon$. Explicit expressions for these quantities in terms of the scalar functions $A$ and $B$ and their derivatives, evaluated on shell are given in the Appendix.

The propagation of the particle in the external field can also be described by an effective interaction appropriate to a Schrödinger description for the propagation of the composite particle. The resolvent of the effective Schrödinger equation is

$$
\tilde{G}_{V}(p)=\frac{Z_{2}^{\prime}}{p^{0}-\epsilon-\mathscr{V}_{S}(p)}+R
$$

where the renormalization constant, $Z_{2}^{\prime}$, the effective interaction, $\mathscr{T}_{S}(p)$, and the remainder function, $R$, are functions of the external field $V$ but independent of $p^{0}$ (they are evalu- 
ated at $p^{0}=\epsilon$ ). The function $R$ describes the propagation of excited states with invariant masses greater than $M$.

For a point particle, the discussion in Sec. II shows that, to order $V^{3}, \mathscr{V}_{S}(p)=V$; there is no effective interaction of order $V^{2}$. The low-energy theorem tells us that this is also true for a composite particle. To prove this, we will first (i) express the effective interaction $\mathscr{V}_{S}(p)$ in terms of the vertex function $\Lambda^{0}(p, p)$ and the contact term $C^{00}(p, 0)$, and then (ii) show that $\mathscr{T}_{S}(p)=V$.
To carry out step (i), expand the propagator (3.13) to second order in $V^{2}$, giving:

$$
\tilde{G}_{V}(p) \simeq \frac{Z_{2}^{\prime}}{p^{0}-\epsilon}+\frac{Z_{2}^{\prime} \mathscr{V}_{S}(p)}{\left(p^{0}-\epsilon\right)^{2}}+\frac{Z_{2}^{\prime} \mathscr{V}_{S}^{2}(p)}{\left(p^{0}-\epsilon\right)^{3}}+\cdots+R
$$

Substituting the expansions (3.7) and (3.12) into (3.10) gives a similar expansion for $G_{V}(p)$ :

$$
\begin{aligned}
G_{V}= & G_{0}+\delta g+V\left[G_{0} \Lambda_{0}^{0} G_{0}+\delta g \Lambda_{0}^{0} G_{0}+G_{0} \Lambda_{0}^{0} \delta g+\left(p^{0}-\epsilon\right) G_{0} \delta \Lambda_{0} G_{0}\right]+V^{2}\left\{G_{0} \Lambda_{0}^{0} G_{0} \Lambda_{0}^{0} G_{0}+\frac{1}{2} G_{0} C_{0}^{00} G_{0}\right. \\
& \left.+\delta g \Lambda_{0}^{0} G_{0} \Lambda_{0}^{0} G_{0}+G_{0} \Lambda_{0}^{0} \delta g \Lambda_{0}^{0} G_{0}+G_{0} \Lambda_{0}^{0} G_{0} \Lambda_{0}^{0} \delta g+\left(p^{0}-\epsilon\right)\left[G_{0} \delta \Lambda_{0} G_{0} \Lambda_{0}^{0} G_{0}+G_{0} \Lambda_{0}^{0} G_{0} \delta \Lambda_{0} G_{0}\right]+\frac{X}{p^{0}-\epsilon}\right\} \\
& +\mathcal{Q}\left(p^{0}-\epsilon\right),
\end{aligned}
$$

where all terms of order $V^{3}$ are neglected, and the terms involving $\delta g$ and $X$ do not affect the result to be proved. Projecting out the positive energy part of Eq. (3.15) and equating powers of $p^{0}-\epsilon$ to similar powers in the expansion of $\tilde{G}_{V}(p)$,

$$
\bar{u}(p) \gamma^{0} G_{V}(p) \gamma^{0} u(p)=\tilde{G}_{V}(p),
$$

permits us to determine $Z_{2}^{\prime}$ and $\mathscr{V}_{S}(p)$ :

$$
\begin{aligned}
Z_{2}^{\prime}=Z_{2}\left[1+V \bar{\Gamma} \delta \Lambda_{0} \Gamma+V\left(\bar{u} \gamma^{0} \delta g \Lambda_{0}^{0} u+\bar{u} \Lambda_{0}^{0} \delta g \gamma^{0} u\right)\right] \\
+V^{2} X, \\
Z_{2}^{\prime} \mathscr{V}_{S}=Z_{2} V \bar{\Gamma} \Lambda_{0}^{0} \Gamma+\frac{1}{2} Z_{2} V^{2} \bar{\Gamma} C_{0}^{00} \Gamma \\
+Z_{2} V^{2}\left\{\left(\bar{u} \gamma^{0} \delta g \Lambda_{0}^{0} u\right)\left(\bar{\Gamma} \Lambda_{0}^{0} \Gamma\right)+\left(\bar{\Gamma} \Lambda_{0}^{0} \Gamma\right)\right. \\
\quad \times\left(\bar{u} \Lambda_{0}^{0} \delta g \gamma^{0} u\right)+\bar{\Gamma} \Lambda_{0}^{0} \delta g \Lambda_{0}^{0} \Gamma+\left(\bar{\Gamma} \delta \Lambda_{0} \Gamma\right)\left(\bar{\Gamma} \Lambda_{0}^{0} \Gamma\right) \\
\left.+\left(\bar{\Gamma} \Lambda_{0}^{0} \Gamma\right)\left(\bar{\Gamma} \delta \Lambda_{0} \Gamma\right)\right\},
\end{aligned}
$$

$$
Z_{2}^{\prime} \mathscr{V}_{S}^{2}=Z_{2} V^{2}\left(\bar{\Gamma} \Lambda_{0}^{0} \Gamma\right)^{2}
$$

Hence, to first order

$$
\begin{aligned}
\left(Z_{2}^{\prime}\right)^{-1}= & \left(Z_{2}\right)^{-1}\left[1-V\left(\bar{\Gamma} \delta \Lambda_{0} \Gamma+\bar{u} \gamma^{0} \delta g \Lambda_{0}^{0} u\right.\right. \\
& \left.\left.+\bar{u} \Lambda_{0}^{0} \delta g \gamma^{0} u\right)\right]+\mathcal{Q}\left(V^{2}\right),
\end{aligned}
$$

and it follows that the second of Eqs. (3.17) gives

$$
\begin{aligned}
\mathscr{T}_{S}= & V\left(\bar{\Gamma} \Lambda_{0}^{0} \Gamma\right)\left[1-V\left(\bar{\Gamma} \delta \Lambda_{0} \Gamma+\bar{u} \gamma^{0} \delta g \Lambda_{0}^{0} u+\bar{u} \Lambda_{0}^{0} \delta g \gamma^{0} u\right)\right] \\
& +V^{2}\left[\frac{1}{2} \bar{\Gamma} C_{0}^{00} \Gamma+\left(\bar{u} \gamma^{0} \delta g \Lambda_{0}^{0} u\right)\left(\bar{\Gamma} \Lambda_{0}^{0} \Gamma\right)\right. \\
& +\left(\bar{\Gamma} \Lambda_{0}^{0} \Gamma\right)\left(\bar{u} \Lambda_{0}^{0} \delta g \gamma^{0} u\right)+\bar{\Gamma} \Lambda_{0}^{0} \delta g \Lambda_{0}^{0} \Gamma \\
& +\left(\bar{\Gamma} \delta \Lambda_{0} \Gamma\right)\left(\bar{\Gamma} \Lambda_{0}^{0} \Gamma\right)+\left(\bar{\Gamma} \Lambda_{0}^{0} \Gamma\left(\bar{\Gamma} \delta \Lambda_{0} \Gamma\right)\right.
\end{aligned}
$$

In the Appendix we show that

$$
\begin{gathered}
\bar{\Gamma} \Lambda_{0}^{0} \Gamma=1, \\
\bar{\Gamma} C_{0}^{00} \Gamma=-\bar{\Gamma} \delta \Lambda_{0} \Gamma=\frac{2 \epsilon}{M^{2}} \xi+\frac{\mathbf{p}^{2}}{M^{2} \epsilon}(1-Z),
\end{gathered}
$$

where $\xi$ and $Z$ are given by Eqs. (A4) and (A6). Hence a number of terms cancel in Eq. (3.19), and it reduces to

$$
\mathscr{T}_{S}=V\left[1+V\left(-\frac{1}{2} \bar{\Gamma} C_{0}^{00} \Gamma+\bar{\Gamma} \Lambda_{0}^{0} \delta g \Lambda_{0}^{0} \Gamma\right)\right] .
$$

Note that the first-order term in $\mathscr{V}_{S}$ is simply $V$, as expected. We wish to emphasize that the second-order term, $V^{2}$, involves a matrix element of $\delta g$, the difference of the full propagator, $G$ and the positive energy propagator $G_{0}$, as defined in Eq. (3.7). Evaluation of this matrix element in the Appendix gives

$$
\bar{\Gamma} \Lambda_{0}^{0} \delta g \Lambda_{0}^{0} \Gamma=\frac{1}{2}\left[\frac{2 \epsilon}{M^{2}} \xi+\frac{\mathbf{p}^{2}}{M^{2} \epsilon}(1-Z)\right],
$$

and thus the $V^{2}$ term is zero, independent of the details of the structure of the bound state. Note that the third of the Eqs. (3.17) is also consistent with $\mathscr{V}_{S}=V$.

This analysis establishes in a straightforward way the factors which enter the calculation of the second-order interaction and Eq. (3.14) illustrates an important point. The two potential terms in Eq. (3.14) may be combined and each 
expanded up to second order in powers of the external interaction strength $V$. Omitting extraneous factors, the potential terms in the expansion of $\tilde{G}_{V}$ are,

$$
\mathscr{T}_{S}^{(1)}(p)+\mathscr{T}_{S}^{(2)}(p)+\frac{\left(\mathscr{T}_{S}^{(1)}(p)\right)^{2}}{p^{0}-\epsilon}+\cdots,
$$

which shows that the second-order terms in $\tilde{G}_{V}$ contain the true second-order interaction $\mathscr{T}_{S}^{(2)}$ that is irreducible with respect to intermediate propagations of the form $\left(p^{0}-\epsilon\right)^{-1}$, plus the iterated first-order interaction evaluated using the on-mass-shell vertex, $\Lambda_{0}^{0}$. The term involving $\left(\mathscr{T}_{S}^{(1)}\right)^{2} /\left(p^{0}-\epsilon\right)$ must be subtracted from the result of expanding $\tilde{G}_{V}$ in order to isolate the irreducible potential. In Sec. $V$ we will use this fact in a slightly different demonstration of the low energy theorem.

To generalize the analysis we now develop a suitable scalar interaction.

\section{SCALAR INTERACTION CORRESPONDING TO A PURE MASS SHIFT}

Construction of a scalar interaction that generates a pure shift of the mass of the composite system provides an illustration of the general arguments of Sec. II. In the bubble model, the scalar interaction is determined by scaling all parameters in the Lagrangian with dimensions of mass to new values $m \rightarrow \lambda m, \mu \rightarrow \lambda \mu, g \rightarrow \lambda^{-1} g$, and similarly scaling all cutoff masses associated with $\mathscr{L}_{\Lambda}$, such that the bound state mass $M$ is scaled to $\lambda M$. Choosing $\lambda=1+S / M$ produces the desired shift of the mass and it implies that the Lagrangian, which includes interactions of the scalar field $S$, has the form (to first order in $S$ )

$$
\mathscr{L} \rightarrow \mathscr{L}-\rho S
$$

The scale-breaking charge associated with the mass scaling in the bubble model is

$$
\begin{aligned}
\rho= & (m / M) \bar{\psi} \psi+2(\mu / M) \phi^{2}-(g / M) \bar{\psi} \psi \phi^{2}-2(\Lambda / M) \phi_{\Lambda}^{2} \\
& +(g / M) \bar{\psi} \psi \phi_{\Lambda}^{2} .
\end{aligned}
$$

The last two terms are necessitated by the cutoff and they correspond to anomalous contributions in a renormalizable theory.

A suitable vector interaction for the bubble model is added to arrive at

$$
\mathscr{B} \rightarrow \mathscr{B}-\rho S-\bar{\psi} \gamma^{0} \psi V
$$

The vertex functions and contact terms associated with this vector interaction are those already defined in Sec. III. It is straightforward to see from $\mathscr{B}$ that the effect of this vector interaction is to shift the time-component of momentum of the constituent fermion. It follows that $\Sigma(p) \rightarrow \Sigma(\tilde{p})$, where $\tilde{p}=\left(p^{0}-V, \mathbf{p}\right)$ and thus the propagator pole is at $\tilde{p}^{2}=M^{2}$, corresponding to $p^{0}=\epsilon+V$. In the previous section we showed that there was no contribution to the second-order interaction from such a vector coupling.

The Ward identity for the scalar vertex follows from examination of the lowest order Feynman diagrams in our simple model. The vertex function corresponding to insertion of the charge $\rho$, in the limit where $q=p^{\prime}-p \rightarrow 0$, is

$$
\Lambda^{S}(p, p)=D_{\rho} \Sigma(p)
$$

where

$$
D_{\rho} \equiv \frac{m}{M} \frac{\partial}{\partial m}+\frac{\mu}{M} \frac{\partial}{\partial \mu}-\frac{g}{M} \frac{\partial}{\partial g}+\frac{\Lambda}{M} \frac{\partial}{\partial \Lambda}
$$

For example, the $(m / M) \bar{\psi} \psi$ term in the charge $\rho$ generates a vertex insertion in the fermion propagator within $\Sigma(p)$, which is the same result as is obtained from $(m / M) \partial \Sigma / \partial m$. Similarly, the $2(\mu / M) \phi^{2}$ term generates a vertex insertion in the scalar meson propagator, which is equivalent to $(\mu / M) \partial \Sigma / \partial \mu$, and so on.

The self-energy, $\Sigma$, is dimensionless, and hence invariant when all parameters with the dimensions of mass are scaled, i.e.,

$$
\Sigma\left(m, \mu, \Lambda, g, p_{\alpha}\right)=\Sigma\left(\lambda m, \lambda \mu, \lambda \Lambda, \lambda^{-1} g, \lambda p_{\alpha}\right)
$$

This invariance provides a series of identities that simplify the analysis. It may be restated as a power series in $\lambda-1$, the first few terms of which are,

$$
\begin{aligned}
\Sigma= & \Sigma+(\lambda-1)\left(D_{\rho}+\frac{p_{\mu}}{M} \frac{\partial}{\partial p_{\mu}}\right) \Sigma+\frac{1}{2}(\lambda-1)^{2} \\
& \times\left(D_{\rho}+\frac{p_{\mu}}{M} \frac{\partial}{\partial p_{\mu}}\right)\left(D_{\rho}+\frac{p_{\nu}}{M} \frac{\partial}{\partial p_{\nu}}\right) \Sigma+\cdots .
\end{aligned}
$$

The derivatives $D_{\rho}+p_{\mu} / M \partial / \partial p_{\mu}$ provide an equivalent form of $d / d \lambda$. From uniqueness of a power series, it follows that the coefficient of each power of $\lambda-1$ vanishes. The vanishing of the coefficient of $\lambda-1$ provides the first identity,

$$
D_{\rho} \Sigma=-\frac{p_{\mu}}{M} \frac{\partial}{\partial p_{\mu}} \Sigma
$$

and the vanishing of the coefficient of $(\lambda-1)^{2}$ provides the second,

$$
D_{\rho}^{2} \Sigma=\frac{p_{\mu}}{M} \frac{\partial}{\partial p_{\mu}} \frac{p_{\nu}}{M} \frac{\partial}{\partial p_{\nu}} \Sigma
$$

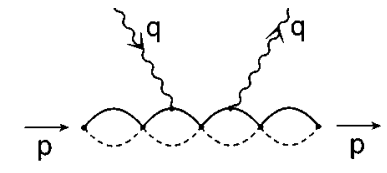

(a)

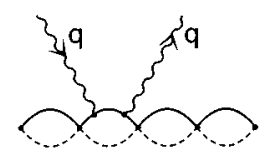

(c)

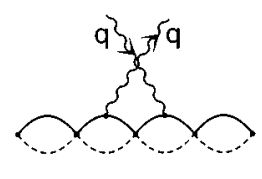

(b)

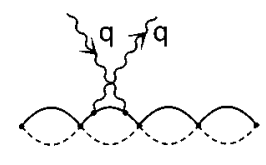

(d)
FIG. 2. Diagramatic representation of the three contributions to Eq. (5.6). 
where we have used Eq. (4.8) to eliminate cross terms involving $2\left(p_{\mu} / M\right) \partial / \partial p_{\mu} D_{\rho} \Sigma$.

Equation (4.8) together with Eq. (4.4) provides a Ward identity for the scalar vertex of the form

$$
\Lambda^{S}(p, p)=-\frac{p_{\mu}}{M} \frac{\partial}{\partial p_{\mu}} \Sigma(p)=-\frac{p}{M} \Sigma^{\prime}(p)
$$

This is a direct consequence of a Ward identity for the divergence of the dilatation current [15] and the particular lowenergy theorem we consider depends on it. Note that the arguments may be applied to the general propagator of Eq. (3.1) and Ward identities of the form of Eqs. (4.8) and (4.9) hold generally.

We now have the necessary equipment to derive the second-order interaction potential for an arbitrary linear combination of scalar plus vector fields. This is done in the next section.

\section{CALCULATION OF SECOND-ORDER INTERACTION}

In Sec. III we calculated the effective first- and secondorder interaction potentials for a vector interaction by examining the response function of the composite system. In this section we will calculate the effective second-order interaction potential for an arbitrary linear combination of vector and scalar fields using an approach based on the Feynman diagrams of the bubble model introduced in Sec. III. While the details of this calculation are somewhat different from those worked out in Sec. III, the two methods are similar and give identical results in the limit of zero momentum transfer. The Feynman diagrams provide the extension to finite momentum transfer. Comparison of the two methods gives added insight into the physics.

Feynman graph analysis requires the calculation of all "Compton" scattering graphs which include absorption and emission of either a vector field $V$ or a scalar field $S$. The "strong" interactions, which depend on the coupling strength $g$, are summed to all orders by summing all bubble graphs of the type shown in Fig. 2. There are three elementary types of bubbles: the self-energies $\Sigma(p)$, the vertex graphs $\Lambda^{S V}(p, p+q)$ which describe the absorption of a field quanta (either $S$ or $V$ ) with four-momentum $q$, and "contact" interactions $C^{S V}(p, q)$ which describe the absorption and emission of an $S$ or $V$ quanta from within a single bubble. For derivation of the low-energy theorem it is sufficient to limit ourselves to forward scattering in which the initial and final bound state four-momenta are both $p$, and the incoming and outgoing field quanta both have four-momenta $q$; the same restriction will apply to the contact terms $C^{S V}$. The summation of the self-energy bubble graphs $\Sigma(p)$ generates the bound state, as already described in Sec. III.

The vertex which describes the absorption of either a scalar or a vector quanta has the form

$$
\Lambda^{S V}(p, p+q)=S \Lambda^{S}(p, p+q)+V \Lambda^{0}(p, p+q) .
$$

When $q=0$, the vertex function $\Lambda^{S}(p, p)$ is given in Eq. (4.10), and the function $\Lambda^{0}(p, p)$ in Eq. (3.12). Similarily, the contact term $C^{S V}$ has the form

$$
C^{S V}(p, q)=S^{2} C^{S S}(p, q)+2 S V C^{0 S}(p, q)+V^{2} C^{00}(p, q) .
$$

The $C^{00}$ contact term for $q=0$ is given in Eq. (3.12). For scalar interactions, the contact term $C^{S S}$ corresponds to two insertions of $\rho$. If we write $D_{\rho}$ in the form $D_{\rho}=\Sigma_{i}\left(m_{i} / M\right) \partial / \partial m_{i}$, where $\left\{m_{i}\right\}=\left\{m, \mu, \Lambda, g^{-1}\right\}$, then at $q=0$ the contact term is given by

$$
\begin{aligned}
C^{S S}(p, 0) & =\sum_{i} \sum_{j} \frac{m_{i} m_{j}}{M^{2}} \frac{\partial^{2} \Sigma(p)}{\partial m_{i} \partial m_{j}}, \\
& =\left(D_{\rho}^{2}-\frac{D_{\rho}}{M}\right) \Sigma(p) .
\end{aligned}
$$

The second partial derivatives in this expression that involve the fermion mass $m$ correspond to two insertions of $(m / M) \bar{\psi} \psi$, the first generating a vertex function connected to two fermion propagators. The second $\partial / \partial m$ generates vertex insertions in each of the two fermion propagators. The other terms in $D_{\rho}^{2}-D_{\rho} / M$ generate in a similar fashion all the rest of the required contributions.

The scalar contact terms may be rewritten using the identities of Eqs. (4.8) and (4.9), as follows,

$$
\begin{aligned}
C^{S S}(p, 0) & =\frac{p_{\mu}}{M} \frac{\partial}{\partial p_{\mu}} \frac{p_{\nu}}{M} \frac{\partial}{\partial p_{\nu}} \Sigma(p)+\frac{p_{\mu}}{M^{2}} \frac{\partial \Sigma(p)}{\partial p_{\mu}} \\
& =\frac{2 p}{M^{2}} \Sigma^{\prime}(p)+\Sigma^{\prime \prime}(p) .
\end{aligned}
$$

When $p=M, \Sigma^{\prime}=\Sigma_{0}^{\prime}$, and $\Sigma^{\prime \prime}=\Sigma_{0}^{\prime \prime}$. There are also cross terms involving one $\rho$ and one vector insertion. For $q=0$ these are

$$
\begin{aligned}
C^{0 S}(p, 0) & =\frac{\partial}{\partial p_{0}} \frac{p_{\mu}}{M} \frac{\partial \Sigma(p)}{\partial p_{\mu}} \\
& =\frac{\gamma^{0}}{M} \Sigma^{\prime}(p)+\frac{p^{0}}{M} \Sigma^{\prime \prime}(p)+2\left(\frac{p \gamma^{0}}{M}-\frac{p^{0}}{M}\right) B^{\prime}\left(p^{2}\right),
\end{aligned}
$$

where the last term will not contribute to the matrix element of interest. The contact term in which the order of the scalar and vector insertions is switched yields the same result.

In terms of the elementary bubbles defined above, the effective second-order interaction, derived from the graphs shown in Fig. 2, can be written: 


$$
\begin{aligned}
\mathscr{T}^{(2)}(p)= & \lim _{q \rightarrow 0} \mathscr{T}^{(2)}(p, q)=\frac{1}{2} \bar{\Gamma}(p)\left\{\Lambda^{S V}(p, p+q) G(p+q) \Lambda^{S V}(p+q, p)+\Lambda^{S V}(p, p-q) G(p-q) \Lambda^{S V}(p-q, p)\right. \\
& \left.+C^{S V}(p, q)\right\} \Gamma(p)-\left[\mathscr{V}^{(1)}(p, \bar{p}+q) \frac{1}{\epsilon_{p}+q^{0}-\epsilon_{p+q}} \mathscr{V}^{(1)}(\bar{p}+q, p)\right],
\end{aligned}
$$

where the external momentum is chosen to be on the mass shell, i.e., $p^{2}=M^{2}$. This is important: the low-energy theorem pertains to the on-mass-shell amplitude that arises in a physical scattering process. The first term in the curly braces is the direct pole term, Fig. 2(a), the second term with $q \rightarrow-q$ is the crossed pole term, Fig. 2(b), and the third, contactlike term, Fig.2(c) describes processes involving scattering from the constituents within a single self-energy bubble. The appropriate subtraction of the iteration of the first-order interaction is based on matrix elements between on-mass-shell states, i.e., $\bar{p}+q$ denotes the four-vector $\left(\boldsymbol{\epsilon}_{p+q}, \mathbf{p}+\mathbf{q}\right)$ where $\boldsymbol{\epsilon}_{p+q}=\left[(\mathbf{p}+\mathbf{q})^{2}+M^{2}\right]^{1 / 2}$. The firstorder interaction, $\mathscr{V}^{(1)}$, is

$$
\mathscr{T}^{(1)}(p, \overline{p+q})=\bar{\Gamma}(p) \Lambda^{S V}(p, \overline{p+q}) \Gamma(\overline{p+q})
$$

The low-energy limit $q \rightarrow 0$ must be taken with care, as discussed below.

The contributions of Eq. (5.6) will be divided into four parts as follows:

$$
\mathscr{T}^{(2)}=\mathscr{V}_{Z}^{(2)}+\mathscr{T}_{\delta G}^{(2)}+\mathscr{V}_{\delta \Lambda}^{(2)}+\mathscr{T}_{C}^{(2)}
$$

The first term involves on-mass-shell matrix elements of the vertices, the pole part of the propagator and the subtraction terms as follows,

$$
\begin{aligned}
\mathscr{T}_{Z}^{(2)}(p)= & \lim _{q \rightarrow 0} \frac{1}{2} \bar{\Gamma}(p)\left\{\Lambda^{S V}(p, \overline{p+q}) \frac{Z_{2}}{p+q-M} \Lambda^{S V}(\overline{p+q}, p)+(q \rightarrow-q)\right\} \Gamma(p) \\
& -\lim _{q \rightarrow 0}\left[\mathscr{V}^{(1)}(p, \overline{p+q}) \frac{1}{p^{0}+q^{0}-\epsilon_{p+q}} \mathscr{Y}^{(1)}(\overline{p+q}, p)\right] .
\end{aligned}
$$

The second term involves propagation in excited states of the system, for which the limit $q \rightarrow 0$ gives

$$
\mathscr{T}_{\delta G}^{(2)}(p)=\bar{\Gamma}(p)\left\{\Lambda^{S V}(p, p) Z_{2} \delta G(p) \Lambda^{S V}(p, p)\right\} \Gamma(p) .
$$

The third term involves an expansion of the vertices about the on-mass-shell momenta, which is necessary because of the singularity of the denominator at $q \rightarrow 0$

$$
\begin{aligned}
\mathscr{V}_{\delta \Lambda}^{(2)}(p)= & \lim _{q \rightarrow 0}(p+q-(\overline{p+q}))_{\mu} \frac{1}{2} \bar{\Gamma}(p)\left\{\left.\frac{\partial}{\partial p_{\mu}^{\prime}} \Lambda^{S V}\left(p, p^{\prime}\right)\right|_{p^{\prime}=\overline{p+q}} G(p+q) \Lambda^{S V} \overline{(p+q}, p\right) \\
& \left.+\left.\Lambda^{S V}(p, \overline{p+q}) G(p+q) \frac{\partial}{\partial p_{\mu}^{\prime}} \Lambda^{S V}\left(p^{\prime}, p\right)\right|_{p^{\prime}=\overline{p+q}}+(q \rightarrow-q)\right\} \Gamma(p) .
\end{aligned}
$$

Finally, the fourth term involves the contact terms, for which the limit $q \rightarrow 0$ poses no problem,

$$
\mathscr{T}_{C}^{(2)}(p)=\frac{1}{2} \bar{\Gamma}(p) C^{S V}(p, 0) \Gamma(p)
$$

Using Eqs. (3.12), (5.4), and (5.5) we find immediately

$$
\begin{aligned}
\mathscr{V}_{C}^{(2)}(p)= & -\frac{1}{\epsilon}\left(S+\frac{\epsilon}{M} V\right)\left[S(1-\xi)-\left(\frac{\epsilon V}{M}\right) \xi\right] \\
& +\left(\frac{\epsilon V}{M}\right)^{2} \frac{\mathbf{p}^{2}}{2 \epsilon^{3}}(1-Z) .
\end{aligned}
$$

Now evaluate $\mathscr{V}_{Z}$. The subtraction removes the contribution from positive-energy intermediate states of the propagator which follows from using,

$$
\frac{Z_{2}}{p+q-M}=\frac{\Gamma(p+q) \bar{\Gamma}(p+q)}{p^{0}+q^{0}-\epsilon_{p+q}}+\frac{\Gamma^{(-)}(p+q) \bar{\Gamma}^{(-)}(p+q)}{p^{0}+q^{0}+\epsilon_{p+q}},
$$

where

$$
\Gamma^{(-)}(p) \equiv Z_{2} v(-p),
$$

and $v(-p)$ is a negative-energy Dirac spinor defined in the Appendix. The positive-energy intermediate state contribution is the only singular part of the expression and once it is 
cancelled, there is no difficulty taking the limit $q \rightarrow 0$. Moreover, this limit does not depend on whether $q^{0} \rightarrow 0$ first or $\mathbf{q} \rightarrow 0$ first. Thus $\mathscr{T}_{Z}^{(2)}$ becomes,

$$
\begin{aligned}
\mathscr{T}_{Z}^{(2)}(p)= & \bar{\Gamma}(p) \Lambda^{S V}(p, p) \\
& \times\left\{\frac{\Gamma^{(-)}(p) \overline{\Gamma^{(-)}}(p)}{2 \epsilon}\right\} \Lambda^{S V}(p, p) \Gamma(p) .
\end{aligned}
$$

This is the composite particle $z$-graph contribution. The calculation proceeds as in the Appendix. Because factors of $\not p$ in $\Lambda^{S V}$ become factors of $M$ when the Dirac equation is used, i.e., $\not \Gamma \Gamma(p)=M \Gamma(p)$, we obtain

$$
\begin{aligned}
\bar{\Gamma}(p) \Lambda^{S V}(p, p) \Gamma^{(-)}(p)= & \bar{u}(p)\left(S+\frac{\epsilon}{M} V\right. \\
& \left.+Z V\left(\gamma^{0}-\frac{\epsilon}{M}\right)\right) v(-p) \\
= & -\left[S+\frac{\epsilon}{M} V(1-Z)\right] \frac{\sigma \cdot \mathbf{p}}{2 \epsilon} .
\end{aligned}
$$

Two factors of $Z_{2}^{1 / 2}$ from the wave functions, $\Gamma$ and $\Gamma^{(-)}$, cancel with a factor $-\Sigma_{0}^{\prime}$ from the vertex function. The result is

$$
\mathscr{T}_{Z}^{(2)}(p)=\left(S+\frac{\epsilon}{M} V(1-Z)\right)^{2} \frac{\mathbf{p}^{2}}{2 \epsilon^{3}}
$$

The purely vector part of this result was already obtained in the Appendix. Note that the $z$-graph contribution for a purely scalar interaction is that expected from the Dirac equation, but the contribution from the vector interaction is not. The $z$-graph contribution of the vector interaction is model dependent because it depends on the factor $Z$. The limit $Z \rightarrow 1$ corresponds to a point particle and in this limit, the vector $z$-graph contribution vanishes, as expected. In general, the $z$-graph contribution for the composite fermion does not yield the same contribution that is obtained for a Dirac particle interacting with equivalent scalar and vector potentials.

Using the expression of Eq. (A7), it is a straightforward matter to evaluate the contribution of the off-shell propagation. The result is

$$
\mathscr{T}_{\delta G}^{(2)}(p)=\left(S+\frac{\epsilon}{M} V\right)^{2} \frac{\xi}{\epsilon}-Z(Z-1)\left(\frac{\epsilon V}{M}\right)^{2} \frac{\mathbf{p}^{2}}{2 \epsilon^{3}}
$$

In order to evaluate the contribution from off-shell vertices, note that $[p+q-(\overline{p+q})]_{\mu}$ has only a time component and it has the form $p^{0}+q^{0}-\epsilon_{p+q}$. This factor cancels the corresponding denominator of the propagator's positiveenergy pole term, thus producing a finite result, and all other terms vanish in the limit $q \rightarrow 0$. This observation and some algebra reduces the required calculation to the form,

$$
\begin{aligned}
\mathscr{T}_{\delta \Lambda}^{(2)}(p)= & \bar{\Gamma}(p)\left(S \Lambda^{S}(p, p)+V \Lambda^{0}(p, p)\right) \Gamma(p) \\
& \times \bar{\Gamma}(p) \frac{d}{d p_{0}}\left(S \Lambda^{S}(p, p)+V \Lambda^{0}(p, p)\right) \Gamma(p),
\end{aligned}
$$

where the derivative with respect to $p_{0}$ combines terms corresponding to action of the derivative first on the left argument and then on the right argument of $\Lambda^{S V}(p, p)$. The required matrix elements are:

$$
\begin{gathered}
\bar{\Gamma}(p)\left(S \Lambda^{S}(p, p)+V \Lambda^{0}(p, p)\right) \Gamma(p)=\frac{M}{\epsilon}\left(S+\frac{\epsilon}{M} V\right) \\
\bar{\Gamma}(p)\left(\frac{d}{d p_{0}} \Lambda^{S}(p, p)\right) \Gamma(p)=\frac{1}{M}(1-2 \xi)
\end{gathered}
$$

and

$$
\bar{\Gamma}(p)\left(\frac{d}{d p_{0}} \Lambda^{0}(p, p)\right) \Gamma(p)=-\frac{1}{M}\left(\frac{2 \epsilon}{M} \xi+\frac{\mathbf{p}^{2}}{M \epsilon} \frac{(1-Z)}{M}\right) .
$$

The result for the contribution from off-shell vertices is therefore

$$
\begin{aligned}
\mathscr{T}_{\delta \Lambda}^{(2)}(p)= & \frac{1}{\boldsymbol{\epsilon}}\left(S+\frac{\epsilon}{M} V\right)\left[S(1-2 \xi)-\left(\frac{\epsilon V}{M}\right)\right. \\
& \left.\times\left(2 \xi+\frac{\mathbf{p}^{2}}{\boldsymbol{\epsilon}^{2}}(1-Z)\right)\right] .
\end{aligned}
$$

Substantial cancellations between the contact terms, the offshell vertex corrections, the off-shell propagation, and the $z$-graph contributions are apparent.

Combining the four parts produces the following result for the second-order interaction,

$$
\mathscr{V}^{(2)}(p, p)=\frac{\mathbf{p}^{2} S^{2}}{2 \epsilon^{3}}
$$

Although the various parts have model-dependent contributions involving $\xi$ or $Z-1$, these all cancel in the end. The simple result that is guaranteed by the low-energy theorem emerges and the second-order interaction is the same as is expected from the Dirac equation with equivalent scalar and vector potentials, or more simply, from a mass shift in the relativistic expression for the energy. There is no suppression of this effect by compositeness at zero momentum transfer.

If there is only a scalar interaction, the result may be stated as follows

$$
\mathscr{T}^{(2)}(p, 0, p)=\frac{\mathbf{p}^{2} S^{2}}{2 \epsilon^{3}}+\frac{S^{2}}{\boldsymbol{\epsilon}}[\xi+(\xi-1)+(1-2 \xi)]=\frac{\mathbf{p}^{2} S^{2}}{2 \epsilon^{3}} .
$$

The contributions to Eq. (5.26) arise as follows: the $\mathbf{p}^{2}$ term from the composite particle $z$ graphs, $\xi$ from the off-shell propagation $\delta G(p), \xi-1$ from the contact terms, and $(1-2 \xi)$ from the off-shell expansion of the vertex functions. 
Cancellations render the overall result independent of the factor $\xi$. This result was given in Ref. [1].

\section{CONCLUSIONS AND DISCUSSION}

The low-energy theorem establishes an equivalence between second-order scattering of a composite spin-1/2 system by scalar plus vector interactions of arbitrary strength and the second-order scattering of a Dirac particle by similar potentials. The basic result is guaranteed by Lorentz invariance. It takes a very simple and model-independent form for the special case of a scalar interaction that shifts the mass linearly, i.e., $M \rightarrow M+S$. Birse [2] has discussed more general scalar and vector interactions and has shown that the momentum-dependent term of Eq. (5.25) is accompanied by a model-dependent scalar term when the interaction causes a nonlinear shift of the mass with respect to the scalar strength, $S$. A similar analysis as the one given here based on the Lagrangian of Eq. (3.3) can provide a demonstration of that result as well. Birse's generalization is important because the scalar interaction that gives rise to a linear mass shift may not be applicable to the interactions of a nucleon. Nevertheless, there is a low-energy theorem for an arbitrary scalar interaction that shows that the momentum-dependent term of Eq. (5.25) is always present. Thus the main contribution that arises from $z$ graphs when a nucleon is treated as a Dirac particle in nuclear scattering and mean-field analyses is a simple consequence of Lorentz invariance and scalar interactions. It is not suppressed by compositeness of the nucleon in the low-energy limit.

In order to emphasize the model independent result, the analysis of this paper has been restricted to the $q \rightarrow 0$ limit for a scalar interaction that provides a linear shift of the mass. The bubble model Feynman graphs may be evaluated numerically for finite $q$. The results are model dependent and they are not pursued in this paper. The general expectation for the $q$ dependence is that each of the contributions discussed in Sec. $\mathrm{V}$ has smooth $q$ dependence. The cancellations which produce the low-energy theorem change slowly with $q$ over a range $q<R^{-1}$, where $R$ is a typical dimension of the composite system. Similar behavior is expected for a nucleon. It suggests that the Dirac equation may be used for interactions which vary slowly over the size of a nucleon, such as those in nuclear scattering or mean fields.

\section{ACKNOWLEDGMENTS}

This work was initiated at a joint CEBAF/INT workshop, September 17-26, 1993. Support by the U.S. Department of Energy under Grants No. DE-FG02-93ER-40762 (S.J.W.) and No. DE-AC05-84ER40150 (F.L.G.) is gratefully acknowledged.

\section{APPENDIX: MATRIX ELEMENTS FOR VECTOR INTERACTIONS}

In this appendix we find the quantities $Z_{2}, \delta g, \Lambda^{0}$, and $C^{00}$ introduced in Sec. III, and evaluate the matrix elements given in Eqs. (3.20) and (3.22).

We begin with the expansion of the propagator given in Eq. (3.5). To obtain the results given in Eq. (3.7) we rationalize the propagator, and expand the scalar functions $A$ and $B$ in a power series in the parameter $p^{2}-M^{2}$ :

$$
G(p)=\frac{A\left(p^{2}\right) p+1-B\left(p^{2}\right)}{\left[1-B\left(p^{2}\right)\right]^{2}-A^{2}\left(p^{2}\right) p^{2}} \simeq \frac{1-B_{0}+A_{0} p+\left(p^{2}-M^{2}\right)\left(A_{0}^{\prime} p-B_{0}^{\prime}\right)+G\left[\left(p^{2}-M^{2}\right)^{2}\right]}{\left[1-B_{0}\right]^{2}-A_{0}^{2} M^{2}-\left(p^{2}-M^{2}\right) c_{0}-\left(p^{2}-M^{2}\right)^{2} c_{1}+G\left[\left(p^{2}-M^{2}\right)^{3}\right]},
$$

where the constants $c_{0}$ and $c_{1}$ are

$$
\begin{aligned}
c_{0}= & 2\left[1-B_{0}\right] B_{0}^{\prime}+A_{0}^{2}+2 M^{2} A_{0} A_{0}^{\prime}, \\
& c_{1}=\left[1-B_{0}\right] B_{0}^{\prime \prime}-\left(B_{0}^{\prime}\right)^{2}+M^{2}\left[A_{0} A_{0}^{\prime \prime}+\left(A_{0}^{\prime}\right)^{2}\right]+2 A_{0} A_{0}^{\prime},
\end{aligned}
$$

with $A_{0}=A\left(M^{2}\right), A_{0}^{\prime}=d A\left(p^{2}\right) /\left.d p^{2}\right|_{p^{2}=M^{2}}, A_{0}^{\prime \prime}=d^{2} A\left(p^{2}\right) /$ $\left.d^{2} p^{2}\right|_{p^{2}=M^{2}}$, and similarly for $B_{0}, B_{0}^{\prime}$, and $B_{0}^{\prime \prime}$. If the propagator is to have a positive energy pole at $p^{2}=M^{2}$, then $1-B_{0}=M A_{0}$, and the propagator reduces to

$$
\begin{aligned}
G(p) \simeq & Z_{2}\left\{\frac{M+\not p}{p^{2}-M^{2}}+\frac{A_{0}^{\prime}}{A_{0}} p-\frac{B_{0}^{\prime}}{A_{0}}-(M+\not p) \frac{c_{1}}{c_{0}}\right\} \\
& +\odot\left(p^{2}-M^{2}\right),
\end{aligned}
$$

where

$$
-\left(Z_{2}\right)^{-1} \equiv A_{0}+2 M\left[M A_{0}^{\prime}+B_{0}^{\prime}\right]=\Sigma_{0}^{\prime}=\left.\frac{d \Sigma(\not p)}{d p}\right|_{p=M},
$$

is a wave function normalization constant. Nonelementary propagation due to excited states of invariant masses greater than $M$ gives rise to the additional term $\delta G(p)$ and $\delta g$ [defined in Eq. (3.7)] which are nonsingular at $p_{0} \rightarrow \epsilon$. Introducing

$$
\begin{aligned}
\Sigma_{0}^{\prime \prime} & =\left.\frac{d^{2} \Sigma(\not)}{d p^{2}}\right|_{p=p} \\
& =4 M^{2}\left(A_{0}^{\prime \prime} M+B_{0}^{\prime \prime}\right)+2\left(A_{0}^{\prime} M+B_{0}^{\prime}\right)+4 M A_{0}^{\prime}
\end{aligned}
$$

and two constants

$$
\xi \equiv M\left(\frac{A_{0}^{\prime} M-B_{0}^{\prime}}{A_{0}}-2 \frac{c_{1}}{c_{0}}\right)=\frac{M}{2} Z_{2} \Sigma_{0}^{\prime \prime}, \quad Z \equiv-Z_{2} A_{0},
$$

we find 


$$
\begin{gathered}
\delta G(p)=\frac{p+M}{2 M^{2}} \xi+\left(\frac{1-Z}{2 Z}\right) \frac{p-M}{2 M^{2}}+\odot\left(p^{2}-M^{2}\right), \\
\delta g=Z_{2}\left[\frac{v(-p) \bar{v}(-p)}{p^{0}+\epsilon}+\delta G(p)\right] .
\end{gathered}
$$

Here $v(-p)$ is a negative-energy Dirac spinor defined by

$$
v(p)=\left(\frac{\boldsymbol{\epsilon}+M}{2 \epsilon}\right)^{1 / 2}\left(\begin{array}{c}
\frac{\sigma \cdot \mathbf{p}}{E_{p}+M} \\
1
\end{array}\right),
$$

and the two constants $\xi$ and $Z$ depend on the internal structure of the composite bound state and are the only constants one needs in order to discuss the various contributions to the second-order interaction.

Next we obtain the expansions given in Eq. (3.12). The vertex function is

$$
\Lambda^{0}(p, p) \equiv-\frac{\partial \Sigma(p)}{\partial p_{0}}=-\left\{\gamma^{0} A_{0}+2 p^{0}\left(A_{0}^{\prime} p+B_{0}^{\prime}\right)+\left(p^{2}-M^{2}\right)\left[\gamma^{0} A_{0}^{\prime}+2 p^{0}\left(A_{0}^{\prime \prime} p+B_{0}^{\prime \prime}\right)\right]\right\}+\mathcal{G}\left[\left(p^{2}-M^{2}\right)^{2}\right] .
$$

Hence $\Lambda_{0}^{0}$ and $\delta \Lambda_{0}$ defined in Eq. (3.12) are

$$
-\Lambda_{0}^{0}=\gamma^{0} A_{0}+2 \epsilon\left(A_{0}^{\prime} \tilde{p}+B_{0}^{\prime}\right), \quad-\delta \Lambda_{0}=4 \epsilon \gamma^{0} A_{0}^{\prime}+2\left(A_{0}^{\prime} \tilde{p}+B_{0}^{\prime}\right)+4 \epsilon^{2}\left(A_{0}^{\prime \prime} \tilde{p}+B_{0}^{\prime \prime}\right),
$$

where $\tilde{p}=(\epsilon, \mathbf{p})$ and $A_{0}^{\prime}$, etc. are the derivatives of the scalar self-energy functions which we encountered before. The contact term is

$$
C_{0}^{00} \equiv \frac{\partial^{2} \Sigma}{\partial p_{0}^{2}}=4 p^{0} \gamma^{0} A_{0}^{\prime}+2\left(A_{0}^{\prime} p+B_{0}^{\prime}\right)+4\left(p^{0}\right)^{2}\left(A_{0}^{\prime \prime} p+B_{0}^{\prime \prime}\right)+\mathcal{Q}\left(p^{2}-M^{2}\right)
$$

Hence $C_{0}^{00}$ is

$$
C_{0}^{00}=4 \epsilon \gamma^{0} A_{0}^{\prime}+2\left(A_{0}^{\prime} \tilde{p}+B_{0}^{\prime}\right)+4 \epsilon^{2}\left(A_{0}^{\prime \prime} \tilde{p}+B_{0}^{\prime \prime}\right)
$$

We see immediately that

$$
\begin{gathered}
-\bar{\Gamma} \Lambda_{0}^{0} \Gamma=Z_{2} \bar{u}(p)\left[\gamma^{0} A_{0}+2 \epsilon\left(A_{0}^{\prime} M+B_{0}^{\prime}\right)\right] u(p)=1, \\
-\bar{\Gamma} \delta \Lambda_{0} \Gamma=\bar{\Gamma} C_{0}^{00} \Gamma=Z_{2} \bar{u}(p)\left[4 \epsilon \gamma^{0} A_{0}^{\prime}+2\left(A_{0}^{\prime} M+B_{0}^{\prime}\right)+4 \epsilon^{2}\left(A_{0}^{\prime \prime} M+B_{0}^{\prime \prime}\right)\right] u(p) \\
=Z_{2}\left[4 \epsilon A_{0}^{\prime}+\frac{2 M}{\epsilon}\left(A_{0}^{\prime} M+B_{0}^{\prime}\right)+4 M \epsilon\left(A_{0}^{\prime \prime} M+B_{0}^{\prime \prime}\right)\right]=\frac{\epsilon}{M} Z_{2} \Sigma_{0}^{\prime \prime}-\frac{2 \mathbf{p}^{2}}{M \epsilon} Z_{2}\left(A_{0}^{\prime} M+B_{0}^{\prime}\right)=\frac{2 \epsilon}{M^{2}} \xi+\frac{\mathbf{p}^{2}}{M^{2} \epsilon}(1-Z) .
\end{gathered}
$$

Finally, we evaluate the matrix element of $\delta g$. This consists of two terms. The first is the $z$-graph contribution of the composite system, which requires the matrix elements

$$
\bar{u}(p) \Lambda_{0}^{0} v(-p)=\bar{v}(-p) \Lambda_{0}^{0} u(p)=2 \sigma \cdot \mathbf{p}\left(A_{0}^{\prime} M+B_{0}^{\prime}\right) .
$$

Hence the $z$-graph contribution becomes, as $p^{0} \rightarrow \epsilon$,

$$
\langle z \text {-graph }\rangle=Z_{2}^{2} \frac{\bar{u}(p) \Lambda_{0}^{0} v(-p) \bar{v}(-p) \Lambda_{0}^{0} u(p)}{p^{0}+\epsilon} \rightarrow \frac{2 \mathbf{p}^{2}}{\epsilon} Z_{2}^{2}\left(A_{0}^{\prime} M+B_{0}^{\prime}\right)^{2}=\frac{\mathbf{p}^{2}}{2 M^{2} \epsilon}(1-Z)^{2} .
$$

Note that this depends on the value of $Z$, as discussed in Sec. V. The $\delta G$ term gives

$$
\begin{aligned}
\left\langle Z_{2} \delta G\right\rangle & =Z_{2}^{2} \bar{u}(p)\left[\gamma^{0} A_{0}+2 \epsilon\left(A_{0}^{\prime} M+B_{0}^{\prime}\right)\right]\left[\frac{p+M}{2 M^{2}} \xi+\left(\frac{1-Z}{2 Z}\right) \frac{p-M}{2 M^{2}}\right]\left[\gamma^{0} A_{0}+2 \epsilon\left(A_{0}^{\prime} M+B_{0}^{\prime}\right)\right] u(p) \\
& =\frac{\epsilon}{M^{2}} \xi+\frac{\mathbf{p}^{2}}{2 M^{2} \epsilon} Z(1-Z),
\end{aligned}
$$

where we used the identities 


$$
\begin{gathered}
\bar{u}(p) \gamma^{0}(M-\not p) \gamma^{0} u(p)=\bar{u}(p)\left(M+\not p-2 \epsilon \gamma^{0}\right) u(p)=-\frac{2 \mathbf{p}^{2}}{\epsilon}, \\
\bar{u}(p) \gamma^{0}(M+\not p) \gamma^{0} u(p)=\bar{u}(p)\left(M-\not p+2 \epsilon \gamma^{0}\right) u(p)=2 \epsilon .
\end{gathered}
$$

Combining (A15) and (A16) gives Eq. (3.22):

$$
\langle z \text {-graph }\rangle+\left\langle Z_{2} \delta G\right\rangle=\frac{\epsilon}{M^{2}} \xi+\frac{\mathbf{p}^{2}}{2 M^{2} \epsilon}(1-Z) .
$$

[1] S. J. Wallace, F. L. Gross, and J. A. Tjon, Phys. Rev. Lett. 74, 228 (1995).

[2] M. C. Birse, Phys. Rev. C 51, R1083 (1995).

[3] M. C. Birse and S. J. Wallace (unpublished).

[4] S. J. Wallace, Ann. Rev. Part. Nucl. Sci. 36, 267 (1987).

[5] L. Ray, G. W. Hoffmann, and W. R. Coker, Phys. Rep. 212, 223 (1992).

[6] B. C. Clark, L. Mercer, and P. Schwandt, Phys. Lett. 122B, 211 (1983).

[7] J. A. McNeil, J. R. Shepard, and S. J. Wallace, Phys. Rev. Lett. 50, 1439 (1983).

[8] J. R. Shepard, J. A. McNeil, and S. J. Wallace, Phys. Rev. Lett.
50, 1443 (1983).

[9] B. C. Clark, S. Hama, R. L. Mercer, L. Ray, and B. D. Serot, Phys. Rev. Lett. 50, 1643 (1983).

[10] J. A. Tjon and S. J. Wallace, Phys. Rev. C 36, 1085 (1987).

[11] S. J. Brodsky, Comments Nucl. Part. Phys. 12, 213 (1984).

[12] E. Bleszynski, M. Bleszynski, and S. Brodsky, Phys. Rev. Lett. 59, 423 (1987).

[13] T. D. Cohen, Phys. Rev. C 45, 833 (1992).

[14] T. Jaroszewicz and S. J. Brodsky, Phys. Rev. C 43, 1946 (1991).

[15] S. Coleman, Aspects of Symmetry (Cambridge University Press, New York, 1988), p. 67. 